\title{
PRICING AND HEDGING OF QUANTILE OPTIONS IN A FLEXIBLE JUMP DIFFUSION MODEL
}

\author{
NING CAI,* The Hong Kong University of Science and Technology
}

\begin{abstract}
This paper proposes a Laplace-transform-based approach to price the fixed-strike quantile options as well as to calculate the associated hedging parameters (delta and gamma) under a hyperexponential jump diffusion model, which can be viewed as a generalization of the well-known Black-Scholes model and Kou's double exponential jump diffusion model. By establishing a relationship between floating- and fixed-strike quantile option prices, we can also apply this pricing and hedging method to floating-strike quantile options. Numerical experiments demonstrate that our pricing and hedging method is fast, stable, and accurate.

Keywords: Jump diffusion; option pricing; hyperexponential; quantile option; Euler inversion

2010 Mathematics Subject Classification: Primary 60J75
\end{abstract}

Secondary 44A10

\section{Introduction}

A quantile option is a fairly new path-dependent security derivative that was first introduced by Miura [28]. It has a payoff dependent on the $\alpha$-quantile, for some $\alpha \in[0,1]$, of the underlying asset price process during a prespecified time period $[0, T]$, which is defined as the smallest barrier such that the fraction of time spent by the underlying price at or below it during $[0, T]$ exceeds $\alpha$ (see Definition 2.1). Since their advent, quantile options have received much attention from both investors and researchers primarily because they may be regarded as an alternative of many popular exotic options such as barrier, lookback, and Asian options. First, as discussed by Broadie and Detemple [6], a fixed-strike quantile option may serve as a replacement for the standard Barrier option by overcoming its deficiency of leaving the buyer without his or her position at the first cross of the barrier. Second, a floating-strike $\alpha$-quantile put option extends the traditional lookback put option by replacing the running maximum of the underlying asset price process in the payoff with its $\alpha$-quantile. Apparently, the former is reduced to the latter as $\alpha=1$. Third, a special $\alpha$-quantile option when $\alpha=0.5$ behaves similarly to the Asian option due to the close relationship between the median and the mean.

Under the Black-Scholes model (BSM), distributions of the associated quantiles have been studied by Dassios [13] (also see [4], [17], and [34]) and led to analytical solutions to pricing of the fixed-strike quantile options; see [2] and [13]. Leung and Kwok [26] established a relationship between fixed- and floating-strike quantile option prices under the BSM. However, as regards numerical implementation, few papers offer numerical results even under the BSM due to computational intractability of available pricing formulae, which involve multiple integrals. One example is the forward shooting grid method developed by Kwok and Lau [25]. For pricing other options dependent on quantiles or occupation times under the BSM, we refer

Received 8 March 2010; revision received 11 February 2011.

* Postal address: Department of Industrial Engineering and Logistics Management, Room 5521, The Hong Kong University of Science and Technology, Clear Water Bay, Kowloon, Hong Kong. Email address: ningcai@ust.hk 
the reader to, e.g. [15], [18], [19], [21], [27], and [29]. In terms of the literature of quantile option pricing beyond the BSM, see, e.g. the occupation time-based approach in [26] under the constant elasticity of variance (CEV) model and in [9] under Kou's double exponential jump diffusion model (abbreviated to DEM).

In this paper we will investigate pricing and hedging of both fixed-and floating-strike quantile options under a hyperexponential jump diffusion model (abbreviated to HEM) (see, e.g. [8] and [22]), where the jump sizes assume a hyperexponential distribution. As a generalization of the BSM and DEM, the HEM has at least three advantages. First, it can better capture the leptokurtic feature by accounting for the uncertainty about heaviness of asset return tails. Second, it can be used to approximate exponential Lévy models with completely monotone Lévy densities such as the CGMY, NIG, and VG models. Third, it can lead to analytical solutions of many path-dependent options such as lookback, single-barrier, and double-barrier options. In this paper it will be shown that we can also price quantile options and calculate the hedging parameters thanks to the analytical tractability of the HEM.

The contribution of our paper is three-fold.

1. Based on Dassios' remarkable identity in law on quantiles of Lévy processes (see [14]), we develop a Laplace-transform-based method to price the fixed-strike quantile option and to calculate the hedging parameters (delta and gamma) numerically under the HEM. See Section 3.1.

2. By establishing a simple relationship between floating- and fixed-strike quantile option prices under the HEM, we can also price floating-strike quantile options and calculate the hedging parameters in a similar way. See Section 3.2.

3. We implement the pricing and hedging methods by using the Euler inversion algorithm twice. The primary difficulty lies in the fact that in general the Euler inversion algorithm applies only for real original functions, but during the implementation we need to evaluate a complex function by inverting its Laplace transform. We circumvent this difficulty by evaluating the real part and the imaginary part of the complex function by inverting their respective Laplace transforms. See Section 4.2. This numerical pricing method turns out to be fast, stable, and accurate. See Section 5. In particular, under the BSM, this method can be simplified to a single Euler inversion in that in this special case, a closed-form Laplace transform of the quantile option price is available. See Section 3.1.

The rest of the paper is organized as follows. Section 2 introduces the HEM and some preliminaries. In Section 3 we present our main results, including pricing and hedging of the fixed-strike quantile options, and establishing a relationship between floating- and fixed-strike quantile option prices. In Section 4, a pricing and hedging algorithm based on Euler inversion is discussed in detail. Numerical results are provided in Section 5. Most proofs are deferred to Appendices A and B.

\section{Background and preliminaries}

\subsection{The HEM}

Under the HEM, for the asset price $S(t)$, the return process $X(t):=\log (S(t) / S(0))$ under a risk-neutral probability measure $\mathrm{P}$ is given by

$$
X(t)=\mu t+\sigma W(t)+\sum_{i=1}^{N(t)} Y_{i}, \quad X(0)=0 .
$$


Here $\mu=r-\sigma^{2} / 2-\lambda \zeta, \zeta=\mathrm{E}\left[\mathrm{e}^{Y_{1}}\right]-1, r$ is the risk-free interest rate, $\sigma$ is the volatility, $\{N(t): t \geq 0\}$ is a Poisson process with rate $\lambda,\{W(t): t \geq 0\}$ is a standard Brownian motion, and $\left\{Y_{i}: i=1,2, \ldots\right\}$ is a sequence of independent, identically distributed (i.i.d.) hyperexponential random variables with probability density function (PDF)

$$
f_{Y}(x)=\sum_{i=1}^{m} p_{i} \eta_{i} \mathrm{e}^{-\eta_{i} x} \mathbf{1}_{\{x \geq 0\}}+\sum_{j=1}^{n} q_{j} \theta_{j} \mathrm{e}^{\theta_{j} x} \mathbf{1}_{\{x<0\}}
$$

where $p_{i}>0$ and $\eta_{i}>1$ for all $i=1, \ldots, m, q_{j}>0$ and $\theta_{j}>1$ for all $j=1, \ldots, n$, and $\sum_{i=1}^{m} p_{i}+\sum_{j=1}^{n} q_{j}=1$. Here $\eta_{i}>1$ for all $i$ and $\theta_{j}>1$ for all $j$ guarantee finiteness of both $\mathrm{E}\left[\mathrm{e}^{Y_{1}}\right]$ and $\mathrm{E}\left[\mathrm{e}^{-Y_{1}}\right]$. Moreover, it is assumed that $\{N(t)\},\{W(t)\}$, and $\left\{Y_{i}\right\}_{i=1}^{\infty}$ are independent.

We point out that the risk-neutral probability measure $P$ is not unique within the jump diffusion framework due to the incompleteness of the model. However, Kou [23] showed that a particular risk-neutral probability measure $\mathrm{P}$ can be chosen within a rational expectations equilibrium setting. It is called risk neutral because the equilibrium price is given by the expectation under $\mathrm{P}$ of the discounted option payoff.

Note that $\{X(t)\}$ is a Lévy process and its Lévy exponent is given by

$$
G(x):=\frac{\log \left(\mathrm{E}\left[\mathrm{e}^{x X(t)}\right]\right)}{t}=\frac{\sigma^{2}}{2} x^{2}+\mu x+\lambda\left(\sum_{i=1}^{m} \frac{p_{i} \eta_{i}}{\eta_{i}-x}+\sum_{j=1}^{n} \frac{q_{j} \theta_{j}}{\theta_{j}+x}-1\right) .
$$

It can be easily shown (see, e.g. [7]) that the equation $G(x)=s$ for all $s>0$ has exactly $m+n+2$ real roots $\beta_{1, s}, \beta_{2, s}, \ldots, \beta_{m+1, s}, \gamma_{1, s}, \gamma_{2, s}, \ldots, \gamma_{n+1, s}$ that satisfy

$$
\begin{gathered}
-\infty<\gamma_{n+1, s}<-\theta_{n}<\gamma_{n, s}<-\theta_{n-1}<\cdots<\gamma_{2, s}<-\theta_{1}<\gamma_{1, s}<0, \\
0<\beta_{1, s}<\eta_{1}<\beta_{2, s}<\cdots<\eta_{m-1}<\beta_{m, s}<\eta_{m}<\beta_{m+1, s}<+\infty .
\end{gathered}
$$

These roots will be used in the subsequent parts.

\subsection{Running maxima and running minima}

Consider the running maximum and running minimum of the process $X:=\{X(t)\}$ :

$$
M_{X}(t):=\sup _{\{0 \leq s \leq t\}} X(s) \text { and } m_{X}(t):=\inf _{\{0 \leq s \leq t\}} X(s) .
$$

Denote by $\mathcal{L}_{M}(s)$ and $\mathcal{L}_{m}(s)$ the Laplace transforms of $\mathrm{E}\left[\mathrm{e}^{v M_{\chi}(t)}\right]$ and $\mathrm{E}\left[\mathrm{e}^{v m_{X}(t)}\right]$ with respect to (w.r.t.) $t$, respectively. More precisely,

$$
\mathcal{L}_{M}(s):=\int_{0}^{\infty} \mathrm{e}^{-s t} \mathrm{E}\left[\mathrm{e}^{v M_{X}(t)}\right] \mathrm{d} t \quad \text { and } \quad \mathcal{L}_{m}(s):=\int_{0}^{\infty} \mathrm{e}^{-s t} \mathrm{E}\left[\mathrm{e}^{v m_{X}(t)}\right] \mathrm{d} t .
$$

Under the HEM, Cai and Kou [8] derived a closed-form expression of $\mathcal{L}_{M}(s)$ by connecting it with the first passage time distribution. For convenience, we present this result in Proposition 2.1 .

Proposition 2.1. For any $v \in\left(-\infty, \beta_{1}\right)$, we have

$$
\mathcal{L}_{M}(s)=\frac{1}{s}+\frac{v}{s} \sum_{l=1}^{m+1} \frac{d_{l}}{\beta_{l}-v}, \quad s>0,
$$


where $\beta_{1}, \beta_{2}, \ldots, \beta_{m+1}$, namely $\beta_{1, s}, \beta_{2, s}, \ldots, \beta_{m+1, s}$ given in (2.1), are the $m+1$ positive roots of the equation $G(x)=s$ and $d:=\left(d_{1}, d_{2}, \ldots, d_{m+1}\right)^{\top}$ is uniquely determined by the linear system $A d=I$, where $I=(1,1, \ldots, 1)^{\top}$ is an $(m+1)$-dimensional unit vector and $A$ is the $(m+1) \times(m+1)$ nonsingular matrix

$$
A=\left(\begin{array}{cccc}
1 & 1 & \cdots & 1 \\
\frac{\eta_{1}}{\eta_{1}-\beta_{1}} & \frac{\eta_{1}}{\eta_{1}-\beta_{2}} & \cdots & \frac{\eta_{1}}{\eta_{1}-\beta_{m+1}} \\
\frac{\eta_{2}}{\eta_{2}-\beta_{1}} & \frac{\eta_{2}}{\eta_{2}-\beta_{2}} & \cdots & \frac{\eta_{2}}{\eta_{2}-\beta_{m+1}} \\
\vdots & \vdots & \ddots & \vdots \\
\frac{\eta_{m}}{\eta_{m}-\beta_{1}} & \frac{\eta_{m}}{\eta_{m}-\beta_{2}} & \cdots & \frac{\eta_{m}}{\eta_{m}-\beta_{m+1}}
\end{array}\right) .
$$

Noting that $m_{X}(t)=\inf _{\{0 \leq s \leq t\}} X(s)=-\sup _{\{0 \leq s \leq t\}}(-X(s))$ and applying Proposition 2.1, we can also derive a closed-form expression for $\mathcal{L}_{m}(s)$.

Remark 2.1. We point out that using the Wiener-Hopf factorization (see, e.g. [3]), we can express $\mathcal{L}_{M}(s)$ (or $\mathcal{L}_{m}(s)$ ) in another way as products of some terms:

$$
\mathcal{L}_{M}(s)=\frac{1}{s} \frac{\prod_{i=1}^{m+1}\left(-\beta_{i}\right) \prod_{i=1}^{m}\left(\eta_{i}-v\right)}{\prod_{i=1}^{m} \eta_{i} \prod_{i=1}^{m+1}\left(v-\beta_{i}\right)} .
$$

\subsection{Quantile options}

The $\alpha$-quantile of the process $\mathcal{X}$ during the period $[0, t]$ can be defined through the occupation time.

Definition 2.1. Let $\Gamma(x, t, \mathcal{X}):=\int_{0}^{t} \mathbf{1}_{\{X(s) \leq x\}} \mathrm{d} s$ represent the occupation time of the process $\mathcal{X}:=\{X(s): s \geq 0\}$ staying below a fixed barrier $x$ during the period $[0, t]$. Then, for any $\alpha \in[0,1]$, the $\alpha$-quantile of the process $\mathcal{X}$ during the period $[0, t]$ is defined as

$$
Q(\alpha, t, \mathcal{X}):=\inf \left\{x: \frac{\Gamma(x, t, \mathcal{X})}{t}>\alpha\right\} .
$$

Then, under the risk neutral measure $\mathrm{P}$, the prices of fixed- and floating-strike $\alpha$-quantile options at time 0 with maturity $t$ are given by

$$
C_{\alpha}\left(S_{0}, K, t\right)=\mathrm{e}^{-r t} \mathrm{E}\left[\left(S_{0} \mathrm{e}^{Q(\alpha, t, \mathcal{X})}-K\right)^{+}\right]
$$

and

$$
P_{\alpha}\left(S_{0}, t\right)=\mathrm{e}^{-r t} \mathrm{E}\left[\left(S_{0} \mathrm{e}^{Q(\alpha, t, X)}-S_{t}\right)^{+}\right]
$$

respectively. For asset pricing theories, we refer the reader to [24] and [33].

Apparently, the distribution of the $\alpha$-quantile plays a pivotal role for pricing of $\alpha$-quantile options. Dassios [14] provided a remarkable result about the quantile distribution for any process with stationary and independent increments. 
Proposition 2.2. ([14].) Suppose that $\{X(t): t \geq 0\}$ is a process with stationary and independent increments which starts from 0 . Then, for any $\alpha \in(0,1)$,

$$
Q(\alpha, t) \stackrel{\mathrm{D}}{=} \sup _{\{0 \leq s \leq \alpha t\}} X^{(1)}(s)+\inf _{\{0 \leq s \leq(1-\alpha) t\}} X^{(2)}(s),
$$

where $\left\{X^{(1)}(s): s \geq 0\right\}$ and $\left\{X^{(2)}(s): s \geq 0\right\}$ are independent copies of the process $\{X(s): s \geq$ $0\}$.

This celebrated result is useful as it applies to any Lévy process and, hence, also applies to the return process under the HEM. Dassios' result (2.4) will serve as a basis for our approach to pricing and hedging fixed-strike quantile options in the subsequent sections.

\section{Pricing and hedging of quantile options}

\subsection{Pricing and hedging of fixed-strike quantile options}

To price and hedge a fixed-strike quantile option, applying the idea of Carr and Madan [10], we consider taking the Laplace transform of the option price w.r.t. $\bar{k}:=-\log K$. Since $\bar{k}$ can be negative, the associated Laplace transform is a two-sided Laplace transform, meaning that the original function is defined on the whole real line. In order to apply the two-sided Euler inversion algorithm proposed by Petrella [30], we introduce a scaling factor $X>S_{0}$ and rewrite the fixed-strike quantile option price as

$$
C_{\alpha}\left(S_{0}, K, t\right)=\mathrm{e}^{-r t} C_{\alpha}^{*}\left(S_{0}, k, t\right) \quad \text { with } \quad C_{\alpha}^{*}\left(S_{0}, k, t\right):=X \mathrm{E}\left[\left(\frac{S_{0}}{X} \mathrm{e}^{Q(\alpha, t, X)}-\mathrm{e}^{-k}\right)^{+}\right],
$$

where $k:=\log (X / K)$. Here the introduction of the scaling factor $X$ ensures that the Euler Laplace inversion algorithm converges quickly in the two-sided case (see [30]).

Denote by $\mathcal{L}(v)$ the Laplace transform of $C_{\alpha}^{*}\left(S_{0}, k, t\right)$ w.r.t. $k$. More precisely,

$$
\mathcal{L}(v)=\int_{-\infty}^{+\infty} \mathrm{e}^{-v k} C_{\alpha}^{*}\left(S_{0}, k, t\right) \mathrm{d} k=\int_{-\infty}^{+\infty} \mathrm{e}^{-v k} X \mathrm{E}\left[\left(\frac{S_{0}}{X} \mathrm{e}^{Q(\alpha, t, X)}-\mathrm{e}^{-k}\right)^{+}\right] \mathrm{d} k .
$$

According to Proposition 2.2, we can obtain the following theorem.

Theorem 3.1. (Pricing and hedging of fixed-strike quantile options.) For any $v>0$, we have

$$
\mathcal{L}(v)=\frac{S_{0}^{v+1}}{v(v+1) X^{v}} \mathrm{E}\left[\mathrm{e}^{(v+1) M_{X}(\alpha t)}\right] \mathrm{E}\left[\mathrm{e}^{(v+1) m_{X}((1-\alpha) t)}\right] .
$$

In particular, under the BSM, $\mathcal{L}(v)$ has the closed-form expression

$$
\mathcal{L}(v)=\frac{S_{0}^{v+1}}{v(v+1) X^{v}} h(v+1 ; \mu, \alpha t) h(-v-1 ;-\mu,(1-\alpha) t),
$$

where

$$
\begin{aligned}
h(v ; \mu, t):= & \frac{v \sigma^{2}+\mu}{v \sigma^{2}+2 \mu} \exp \left(\frac{v \sigma^{2} t}{2 v \sigma^{2}+4 \mu}\right) \operatorname{Erfc}\left(-\frac{\left(v \sigma^{2}+\mu\right) \sqrt{t}}{\sigma \sqrt{2}}\right) \\
& +\frac{\mu}{v \sigma^{2}+2 \mu} \operatorname{Erfc}\left(\frac{\mu \sqrt{t}}{\sigma \sqrt{2}}\right),
\end{aligned}
$$

and $\operatorname{Erfc}(z):=(2 / \sqrt{\pi}) \int_{z}^{+\infty} \mathrm{e}^{-x^{2}} \mathrm{~d} x$ represents the complementary error function. 
The two common Greeks $\Delta\left(C_{\alpha}\left(S_{0}, K, t\right)\right)$ and $\Gamma\left(C_{\alpha}\left(S_{0}, K, t\right)\right)$ can be calculated as

$$
\begin{aligned}
& \Delta\left(C_{\alpha}\left(S_{0}, K, t\right)\right)=\frac{\partial}{\partial S_{0}} C_{\alpha}\left(S_{0}, K, t\right)=\left.\mathrm{e}^{-r t} \mathcal{L}^{-1}\left(\frac{(v+1) \mathcal{L}(v)}{S_{0}}\right)\right|_{k=\ln (X / K)}, \\
& \Gamma\left(C_{\alpha}\left(S_{0}, K, t\right)\right)=\frac{\partial^{2}}{\partial S_{0}^{2}} C_{\alpha}\left(S_{0}, K, t\right)=\left.\mathrm{e}^{-r t} \mathcal{L}^{-1}\left(\frac{v(v+1) \mathcal{L}(v)}{S_{0}^{2}}\right)\right|_{k=\ln (X / K)},
\end{aligned}
$$

where $\mathcal{L}^{-1}$ denotes the inverse Laplace transform.

Proof. See Appendix A.

Consequently, under the BSM the closed-form Laplace transform $\mathcal{L}(v)$ is available, so applying the Euler inversion algorithm can generate numerical prices and the two Greeks easily. In contrast, under the general HEM, Theorem 3.1 does not provide us with a closedform Laplace transform $\mathcal{L}(v)$ because neither $\mathrm{E}\left[\mathrm{e}^{(v+1) M_{X}(\alpha t)}\right]$ nor $\mathrm{E}\left[\mathrm{e}^{(v+1) m_{X}((1-\alpha) t)}\right]$ have analytical expressions. However, Proposition 2.1 implies that both of them have closed-form Laplace transforms w.r.t. $t$, namely $\mathcal{L}_{M}(s)$ and $\mathcal{L}_{m}(s)$. Accordingly, in principle we can price fixed-strike quantile options and calculate the two Greeks by applying the Laplace inversion algorithm twice. A rough pricing and hedging algorithm is given as follows.

Step 1. Evaluate $\mathrm{E}\left[\mathrm{e}^{(v+1) M_{X}(\alpha t)}\right]$ and $\mathrm{E}\left[\mathrm{e}^{(v+1) m_{X}((1-\alpha) t)}\right]$ by inverting their Laplace transforms numerically. Then we can obtain $\mathcal{L}(v),(v+1) \mathcal{L}(v) / S_{0}$, and $v(v+1) \mathcal{L}(v) / S_{0}^{2}$.

Step 2. Inverting $\mathcal{L}(v),(v+1) \mathcal{L}(v) / S_{0}$, and $v(v+1) \mathcal{L}(v) / S_{0}^{2}$, and then multiplying the results by e ${ }^{-r t}$ yields $C_{\alpha}\left(S_{0}, K, t\right), \Delta\left(C_{\alpha}\left(S_{0}, K, t\right)\right)$, and $\Gamma\left(C_{\alpha}\left(S_{0}, K, t\right)\right)$, respectively.

It is worth mentioning here that by 'applying the Laplace inversion algorithm twice' we mean that a single Laplace inversion algorithm is applied in both step 1 and step 2 . This greatly differs from the so-called 'double Laplace inversion'. Specifically, if the closed-form expression of $\int_{-\infty}^{+\infty} \int_{0}^{+\infty} \mathrm{e}^{-v k} \mathrm{e}^{-w t} C_{\alpha}^{*}\left(S_{0}, k, t\right) \mathrm{d} t \mathrm{~d} k$ is available, it is called the double Laplace transform of $C_{\alpha}^{*}\left(S_{0}, k, t\right)$ w.r.t. $k$ and $t$. Then the double Laplace inversion algorithm applies (see [11]). However, in our case, a closed-form expression of the double Laplace transform of $C_{\alpha}^{*}\left(S_{0}, k, t\right)$ is not available. So we attempt to evaluate $C_{\alpha}^{*}\left(S_{0}, k, t\right)$ by inverting its single Laplace transform $\mathcal{L}(v)$. But

$$
\mathcal{L}(v)=\frac{S_{0}^{v+1}}{v(v+1) X^{v}} \mathrm{E}\left[\mathrm{e}^{(v+1) M_{X}(\alpha t)}\right] \mathrm{E}\left[\mathrm{e}^{(v+1) m_{x}((1-\alpha) t)}\right]
$$

has no closed-form expression, either. To evaluate $\mathcal{L}(v)$, we need to invert the closed-form Laplace transforms of $\mathrm{E}\left[\mathrm{e}^{(v+1) M_{X}(\alpha t)}\right]$ and $\mathrm{E}\left[\mathrm{e}^{(v+1) m_{X}((1-\alpha) t)}\right]$. To summarize, the whole inversion' should be divided into two steps, each of which uses the single Laplace inversion. This is the crucial difference from 'the double Laplace inversion', and we call it 'Laplace inversion twice'.

Nonetheless, when implementing the above algorithm using the Euler inversion algorithm, we encounter some difficulty, primarily because the Euler inversion algorithm applies for only the real original functions and meanwhile the inversion formula involves Laplace transforms in the complex domain. As a result, to invert $\mathcal{L}(v)$ in step 2, we need values of $\mathcal{L}(v)$ for complex $v$. This means that in step 1 , we need to evaluate $\mathrm{E}\left[\mathrm{e}^{(v+1) M_{X}(\alpha t)}\right]$ and $\mathrm{E}\left[\mathrm{e}^{(v+1) m_{X}((1-\alpha) t)}\right]$ for complex $v$ by inverting their Laplace transforms w.r.t. $t$. If we apply the Euler inversion algorithm in step 1 directly, it will fail in that this is equivalent to applying the Euler inversion algorithm in the case of complex original functions. To circumvent this difficulty, we evaluate 
the real parts and the imaginary parts of $\mathrm{E}\left[\mathrm{e}^{(v+1) M_{X}(\alpha t)}\right]$ and $\mathrm{E}\left[\mathrm{e}^{(v+1) m_{X}((1-\alpha) t)}\right]$ separately. For details, see Section 4.

\subsection{Pricing and hedging of floating-strike quantile options}

Compared with pricing and hedging of the fixed-strike quantile option, the floating-strike quantile option seems more intractable because its price not only depends on the quantile but also on the terminal asset value. Nevertheless, we can bridge these two issues by transforming the floating-strike option price to the price of a fixed-strike option by virtue of Girsanov's theorem for jump diffusion processes. This idea stems from Leung and Kwok [26], who established a relationship between fixed- and floating-strike quantile option prices under the BSM.

In this part we just provide some new notation and main results. For the details of the proof, see Appendix B. To facilitate the derivation, we consider a quantile option, where the underlying asset has a dividend with rate $q$. Note that this slight modification does not affect essentially the correctness of Proposition 2.1 and Theorem 3.1. Rewrite the fixed- and floating-strike quantile option prices with maturity $T$ as

$$
C_{\alpha}\left(S_{0}, K, T, \S\right)=\mathrm{e}^{-r T} \mathrm{E}\left[(Q(\alpha, T, \S)-K)^{+}\right]
$$

and

$$
P_{\alpha}\left(S_{0}, T, \delta\right)=\mathrm{e}^{-r T} \mathrm{E}\left[(Q(\alpha, T, \delta)-S(T))^{+}\right],
$$

respectively, where $Q(\alpha, t, \S)$ is the $\alpha$-quantile of the underlying asset price process $\&$ starting from the point $S_{0}$.

Now we introduce two independent processes $\&$ and $\bar{\S}$. The former is defined as

$$
s:=\left\{S(t):=S_{0} \exp \left(\mu t+\sigma W(t)+\sum_{i=1}^{N(t)} Y_{i}\right): t \geq 0\right\}
$$

where all the parameters are the same as those in (2.1) except that $\mu:=r-q-\sigma^{2} / 2-\lambda \zeta$ and $\eta_{i}>2$ for any $i=1,2, \ldots, m$. The latter is given by

$$
\bar{s}:=\left\{\bar{S}(t):=S_{0} \exp \left(\bar{\mu} t+\sigma \bar{W}(t)+\sum_{i=1}^{\tilde{N}(t)} \bar{Y}_{i}\right): t \geq 0\right\},
$$

where $\bar{\mu}=\bar{r}-\bar{q}-\sigma^{2} / 2-\tilde{\lambda} \bar{\zeta}, \bar{r}=q, \bar{q}=r-\lambda \zeta-\tilde{\lambda} \bar{\zeta}, \bar{\zeta}=\mathrm{Ee}^{\bar{Y}_{1}}-1,\{\tilde{N}(t): t \geq 0\}$ is a Poisson process with rate $\tilde{\lambda}:=\lambda(\zeta+1),\{\bar{W}(t): t \geq 0\}$ is a standard Brownian motion, and $\left\{\bar{Y}_{i}: i=1,2, \ldots\right\}$ is a sequence of i.i.d. hyperexponential random variables with PDF

$$
f_{\bar{Y}}(x)=\sum_{i=1}^{n} \bar{p}_{i} \bar{\eta}_{i} \mathrm{e}^{-\bar{\eta}_{i} x} \mathbf{1}_{\{x \geq 0\}}+\sum_{j=1}^{m} \bar{q}_{j} \bar{\theta}_{j} \mathrm{e}^{\bar{\theta}_{j} x} \mathbf{1}_{\{x<0\}},
$$

with

$$
\begin{gathered}
\bar{p}_{i}=\frac{q_{i} \theta_{i}}{(\zeta+1)\left(\theta_{i}+1\right)}>0 \quad \text { and } \quad \bar{\eta}_{i}=\theta_{i}+1>1 \quad \text { for all } i=1, \ldots, n, \\
\bar{q}_{j}=\frac{p_{j} \eta_{j}}{(\zeta+1)\left(\eta_{j}-1\right)}>0 \quad \text { and } \quad \bar{\theta}_{j}=\eta_{j}-1>1 \quad \text { for all } j=1, \ldots, m .
\end{gathered}
$$

Here $\bar{\theta}_{j}>1$ holds for any $j=1, \ldots, m$ due to the assumption that $\eta_{j}>2$ for any $j=$ $1, \ldots, m$. This guarantees that $\mathrm{E}\left(\mathrm{e}^{-\bar{Y}_{1}}\right)$ is finite. Then we have the following theorem. 
Theorem 3.2. (Pricing and hedging of floating-strike quantile options.) With the above notation, the floating-strike quantile option price is

$$
P_{\alpha}\left(S_{0}, T, \S\right)=C_{\alpha}\left(S_{0}, S_{0}, T, \bar{\S}\right)
$$

and the two common Greeks $\Delta\left(P_{\alpha}\left(S_{0}, T, 8\right)\right)$ and $\Gamma\left(P_{\alpha}\left(S_{0}, T, 8\right)\right)$ are given by

$$
\Delta\left(P_{\alpha}\left(S_{0}, T, \S\right)\right):=\frac{\partial}{\partial S_{0}} P_{\alpha}\left(S_{0}, T, \S\right)=C_{\alpha}(1,1, T, \bar{\gamma})
$$

and

$$
\Gamma\left(P_{\alpha}\left(S_{0}, T, \S\right)\right):=\frac{\partial^{2}}{\partial S_{0}^{2}} P_{\alpha}\left(S_{0}, T, \S\right)=0 .
$$

Proof. See Appendix B.

Theorem 3.2 implies that we can price the floating-strike quantile option by pricing a closely related fixed-strike quantile option. In particular, we can price the European lookback put option at time 0 , where the payoff is given by $S_{0} \mathrm{e}^{M x(T)}-S(T)$, and which is actually a special case of the floating-strike $\alpha$-quantile option with $\alpha=1$.

Remark 3.1. In a recent paper [9] Cai et al. investigated pricing and hedging of occupationtime-related options under the DEM, including the fixed-strike quantile options. In comparison, there exist several key differences between our paper and [9]. First, our model is more general and the DEM is only a special case of the HEM. Second, we solve the pricing and hedging problems for both fixed- and floating-strike quantile options, whereas [9] discussed only the fixed-strike case. Third, their pricing method depends on the distribution of the occupation time, which they spent considerable effort deriving. Our approach, however, does not require this technical result.

\section{The pricing and hedging algorithm via Euler inversion}

In financial applications, numerical inversion of Laplace transforms has become increasingly important for it is always much easier to derive a closed-form expression for the Laplace transform of a derivative price than for the price itself. In this paper we will employ the Euler inversion algorithm to invert Laplace transforms numerically, which was proposed in the onesided case by Abate and Whitt [1] and was extended to the two-sided case by Petrella [30]. For a survey on comparison of different transform inversion methods applied in asset pricing, we refer the reader to [12].

\subsection{The Euler inversion algorithm}

When implementing our pricing method proposed in Section 3, we are confronted with two types of Laplace transform. One is one-sided, meaning that the original function is defined on the positive real line, e.g. $\mathscr{L}_{M}(s)$ and $\mathscr{L}_{m}(s)$; while the other is two-sided, meaning that the original function is defined on the whole real line, e.g. $\mathcal{L}(v)$.

Suppose that $\hat{f}(s)$ is a two-sided Laplace transform of a nonnegative function $f(t)$ defined on the whole real line $\mathbb{R}$, i.e. $\hat{f}(s):=\int_{-\infty}^{+\infty} \mathrm{e}^{-s t} f(t) \mathrm{d} t$. Then we have the following inversion formula (see [30]):

$$
f(t)=\frac{\mathrm{e}^{A / 2}}{2 t} \operatorname{Re}\left(\widehat{f}\left(\frac{A}{2 t}\right)\right)+\frac{\mathrm{e}^{A / 2}}{t} \sum_{j=1}^{\infty}(-1)^{j} \operatorname{Re}\left(\widehat{f}\left(\frac{A-2 j \pi \mathrm{i}}{2 t}\right)\right)-e_{d}
$$


Here $\operatorname{Re}(z)$ represents the real part of $z, e_{d}=e_{d}^{+}+e_{d}^{-}$, and

$$
e_{d}^{+}=\sum_{j=1}^{+\infty} \mathrm{e}^{-j A} f((2 j+1) t) \quad \text { and } \quad e_{d}^{-}=\sum_{j=-\infty}^{-1} \mathrm{e}^{-j A} f((2 j+1) t),
$$

where $A$ is an arbitrary positive constant used to control the discretization error $e_{d}$.

If $\hat{f}(s)$ is a one-sided Laplace transform of a nonnegative function $f(t)$ defined on the positive real line $\mathbb{R}^{+}$, i.e. $\hat{f}(s):=\int_{0}^{+\infty} \mathrm{e}^{-s t} f(t) \mathrm{d} t,(4.1)$ and (4.2) still hold except that $e_{d}^{-}$disappears.

Note that there exist three kinds of parameter involved in the implementation of the Euler inversion algorithm. (i) $\left(n_{1}, n_{2}\right)$. During the computation, we have to calculate some alternating series of the form $\sum_{i=0}^{\infty}(-1)^{i} a_{i}$ in expression (4.1). To accelerate the convergence, we adopt the idea of the Euler transformation, which gives a much faster convergence of the infinite sum (see [1]). More precisely, $\sum_{i=0}^{\infty}(-1)^{i} a_{i}$ can be approximated by the finite sum

$$
E(m, n)=\sum_{k=0}^{m} \frac{m !}{k !(m-k) !} 2^{-m} S_{n+k},
$$

where $S_{j}:=\sum_{i=0}^{j}(-1)^{i} a_{i}$. Since there are two transform inversions in two steps when pricing fixed-strike quantile options, the Euler transformation is used twice. We use $\left(n_{1}, m_{1}\right)$ and $\left(n_{2}, m_{2}\right)$ to express parameters involved in the Euler transformation for Euler inversion w.r.t. the scaled strike $k$ and maturity $t$, respectively. According to Abate and Whitt's suggestion (see [1]), we set $m_{1}=n_{1}+15$ and $m_{2}=n_{2}+15$. In practice, we set $n_{1}=n_{2}=90$. (ii) $A_{1}$ and $A_{2}$. These parameters are used to control the discretization error. The empirical setting is $A_{1}=A_{2}=18$. (iii) The scaling factor $X$. In our case, we will select $X$ as 10000 .

The selection of the parameters $A_{1}, A_{2}$, and $X$ does not affect the accuracy, but does affect the convergence, as shown by the author. This issue will be discussed in detail in Sections 5.2-5.4.

\subsection{Calculate $\mathcal{L}(v)$ for complex $v$ via the Euler inversion algorithm}

To invert $\mathcal{L}(v)$ numerically via the Euler inversion algorithm, we require values of $\mathcal{L}(v)$ for complex numbers $v$. Therefore, we need to evaluate $\mathrm{E}\left[\mathrm{e}^{(v+1) M_{X}(\alpha t)}\right]$ and $\mathrm{E}\left[\mathrm{e}^{(v+1) m_{X}((1-\alpha) t)}\right]$ for complex $v$. More generally, we first illustrate how to calculate $\mathrm{E}\left[\mathrm{e}^{v M_{X}(t)}\right]$ and $\mathrm{E}\left[\mathrm{e}^{v m_{X}(t)}\right]$ for complex $v$ by inverting their Laplace transforms $\mathscr{L}_{M}(s)$ and $\mathscr{L}_{m}(s)$ numerically. Note that the Euler inversion algorithm applies for only real original functions. To solve this problem, we express $g(v, t):=\mathrm{E}\left[\mathrm{e}^{v M_{X}(t)}\right]$ as

$$
g(v, t)=g_{\mathrm{R}}(a, b, t)+g_{\mathrm{I}}(a, b, t) \mathrm{i} \quad \text { for } v=a+b \mathrm{i},
$$

where $g_{\mathrm{R}}(a, b, t)$ and $g_{\mathrm{I}}(a, b, t)$ represent the real part and imaginary parts of $g(v)$, respectively, and

$$
g_{\mathrm{R}}(a, b, t)=\mathrm{E}\left[\mathrm{e}^{a M_{X}(t)} \cos \left(b M_{X}(t)\right)\right] \quad \text { and } \quad g_{\mathrm{I}}(a, b, t)=\mathrm{E}\left[\mathrm{e}^{a M_{X}(t)} \sin \left(b M_{X}(t)\right)\right] .
$$

Then in order to calculate $g(v, t) \equiv \mathrm{E}\left[\mathrm{e}^{v M_{X}(t)}\right]$ for complex $v$, it suffices to evaluate $g_{\mathrm{R}}(a, b, t)$ and $g_{\mathrm{I}}(a, b, t)$ via Euler inversion, both of which are real functions so that the Euler inversion algorithm applies. Denote the Laplace transforms of $g_{\mathrm{R}}(a, b, t)$ and $g_{\mathrm{I}}(a, b, t)$ for a complex number $v=a+b$ i w.r.t. $t$ by $\mathcal{L}_{M \mathrm{R}}(a, b, s)$ and $\mathcal{L}_{M \mathrm{I}}(a, b, s)$, respectively. More precisely, for any $s>0$,

$$
\mathcal{L}_{M \mathrm{R}}(a, b, s):=\int_{0}^{\infty} \mathrm{e}^{-s t} g_{\mathrm{R}}(a, b, t) \mathrm{d} t \quad \text { and } \quad \mathcal{L}_{M \mathrm{I}}(a, b, s):=\int_{0}^{\infty} \mathrm{e}^{-s t} g_{\mathrm{I}}(a, b, t) \mathrm{d} t .
$$

Their closed-form expressions are derived in the following theorem. 
Theorem 4.1. For any $s>0, v=a+b \mathrm{i}$, and $a \in\left(-\infty, \beta_{1}\right)$, we have

$$
\mathcal{L}_{M \mathrm{R}}(a, b, s)=\frac{1}{s}\left\{1+\sum_{l=1}^{m+1}\left[\frac{a\left(\beta_{l}-a\right)-b^{2}}{\left(a-\beta_{l}\right)^{2}+b^{2}} d_{l}\right]\right\}
$$

and

$$
\mathscr{L}_{M \mathrm{I}}(a, b, s)=\frac{1}{s} \sum_{l=1}^{m+1} \frac{b \beta_{l} d_{l}}{\left(a-\beta_{l}\right)^{2}+b^{2}},
$$

where $\beta_{1}, \beta_{2}, \ldots, \beta_{m+1}$ and $d=\left(d_{1}, d_{2}, \ldots, d_{m+1}\right)^{\top}$ are the same as in Proposition 2.1.

Proof. The proof is similar to that of Proposition 2.1 of [8] and, thus, is omitted.

Consequently, for complex $v=a+b \mathrm{i}$, by inverting $\mathcal{L}_{M \mathrm{R}}(a+1, b, \alpha s)$ and $\mathcal{L}_{M \mathrm{I}}(a+1, b, \alpha s)$, we can obtain both $g_{\mathrm{R}}(a+1, b, \alpha t)$ and $g_{\mathrm{I}}(a+1, b, \alpha t)$, hence getting $\mathrm{E}\left[\mathrm{e}^{(v+1) M_{X}(\alpha t)}\right]$. As for $\mathrm{E}\left[\mathrm{e}^{(v+1) m_{x}((1-\alpha) t)}\right]$, a similar idea applies. In this way, we can evaluate $\mathcal{L}(v)$ for complex $v$ numerically.

\subsection{The pricing and hedging algorithm}

To summarize, a detailed pricing and hedging algorithm for quantile options under the HEM is given as follows.

Step 1. Evaluate $g_{\mathrm{R}}(a+1, b, \alpha t)$ and $g_{\mathrm{I}}(a+1, b, \alpha t)$ by inverting their Laplace transforms $\mathcal{L}_{M \mathrm{R}}(a+1, b, \alpha s)$ and $\mathcal{L}_{M \mathrm{I}}(a+1, b, \alpha s)$ in (4.3) and (4.4), respectively. Thus, we obtain $\mathrm{E}\left[\mathrm{e}^{(v+1) M_{X}(\alpha t)}\right]$ for complex $v=a+b \mathrm{i}$.

Step 2. Obtain $\mathrm{E}\left[\mathrm{e}^{(v+1) m x((1-\alpha) t)}\right]$ for complex $v$ in a similar way as in step 1. As a result, we have actually obtained $\mathcal{L}(v),(v+1) \mathcal{L}(v) / S_{0}$, and $v(v+1) \mathcal{L}(v) / S_{0}^{2}$ for complex $v$.

Step 3. Price quantile options and calculate the two Greeks, delta and gamma, by inverting $\mathcal{L}(v),(v+1) \mathcal{L}(v) / S_{0}$, and $v(v+1) \mathcal{L}(v) / S_{0}^{2}$, respectively, and by using their values with complex $v$ obtained via step 1 and step 2 .

In particular, under the BSM, the pricing and hedging algorithm can be simplified to only one step, i.e. step 3 , because $\mathcal{L}(v)$ has the closed-form expression in (3.2).

\section{Numerical results}

\subsection{Calculating prices and Greeks under the HEM}

Based on the pricing and hedging algorithm in Section 4.3, Theorem 3.1, Theorem 3.2, and Theorem 4.1, we can price both fixed- and floating-strike quantile options and calculate the associated two Greeks (delta and gamma) under the HEM. Without loss of generality, we focus on a simple case, $m=n=2$, for the hyperexponential distribution given by (2.2), i.e. both the upward and downward jump size distributions are a mixture of two exponential distributions. The corresponding numerical prices, deltas and gammas (denoted by LL price, LL delta, and LL gamma) of fixed- and floating-strike quantile options are given in Table 1 and Table 2. All the computations are completed on a desktop with an Intel ${ }^{\circledR} 2.66 \mathrm{GHz}$ processor. It turns out that all the numerical results stay within the $95 \%$ confidence intervals of Monte Carlo simulation estimates (denoted by MC price, MC delta, and MC gamma). Moreover, for the pricing, the averages of absolute values of absolute and relative errors are 0.661 cents and $0.116 \%$, respectively. Therefore, we draw the conclusion that our pricing algorithm is accurate. In addition, the CPU time to produce one LL price (LL delta or LL gamma) is around 
TABLE 1: Pricing fixed- and floating-strike quantile options. The default parameters are $\alpha=0.5, r=0.05$, $\eta_{1}=\theta_{1}=30, \eta_{2}=\theta_{2}=40, p_{1}=p_{2}=0.3, q_{1}=q_{2}=0.2, S_{0}=100, t=1, A_{1}=A_{2}=18$, $n_{1}=n_{2}=90$, and $X=10000$. The LL price is obtained by using Laplace inversion twice. The MC price is the Monte Carlo simulation estimate obtained by simulating 100000 paths and using 100000 time steps. SE is the associated standard error. $\mathrm{AE}$ and $\mathrm{RE}$ are the absolute and relative errors between LL price and MC price, respectively.

\begin{tabular}{rrrrrrrrr}
\hline \multicolumn{7}{c}{ Pricing fixed-strike quantile options under the HEM } \\
\hline$\lambda$ & $\sigma$ & \multicolumn{1}{c}{$K$} & LL price & MC price & \multicolumn{1}{c}{ SE } & AE & RE (\%) \\
\hline 1 & 0.2 & 90 & 12.51158 & 12.51355 & 0.00722 & -0.00197 & -0.016 \\
& & 100 & 5.73706 & 5.73799 & 0.01154 & -0.00093 & -0.016 \\
& & 110 & 2.12864 & 2.12184 & 0.01122 & 0.00680 & 0.320 \\
\hline 1 & 0.3 & 90 & 13.69527 & 13.69702 & 0.01323 & -0.00175 & -0.013 \\
& & 100 & 7.73121 & 7.73464 & 0.01720 & -0.00343 & -0.044 \\
& & 110 & 4.03886 & 4.03032 & 0.01758 & 0.00854 & 0.212 \\
\hline 3 & 0.2 & 90 & 12.59588 & 12.59849 & 0.00776 & -0.00261 & -0.021 \\
& & 100 & 5.90413 & 5.90702 & 0.01208 & -0.00289 & -0.049 \\
& & 110 & 2.29206 & 2.28497 & 0.01186 & 0.00709 & 0.310 \\
\hline 3 & 0.3 & 90 & 13.77124 & 13.77766 & 0.01357 & -0.00642 & -0.047 \\
& & 100 & 7.84386 & 7.85146 & 0.01754 & -0.00760 & -0.097 \\
& & 110 & 4.15415 & 4.14703 & 0.01796 & 0.00712 & 0.172 \\
\hline \multicolumn{7}{c}{ Pricing floating-strike quantile } & options under the HEM & \\
\hline$\lambda$ & $\sigma$ & $K$ & LL price & MC price & SE & AE & RE $(\%)$ \\
\hline 1 & 0.2 & - & 3.45566 & 3.44620 & 0.01669 & 0.00946 & 0.275 \\
& 0.3 & - & 5.52382 & 5.50849 & 0.02468 & 0.01533 & 0.278 \\
3 & 0.2 & - & 3.62904 & 3.61563 & 0.01739 & 0.01341 & 0.371 \\
& 0.3 & - & 5.64106 & 5.62456 & 0.02510 & 0.01650 & 0.293 \\
\hline
\end{tabular}

21 seconds, which is practically acceptable and is much less than that of the Monte Carlo simulation (around 25 minutes). So our pricing algorithm is also efficient. In particular, we can price both fixed- and floating-strike quantile options under two important models, the BSM and the DEM, in the same way (note that quantile options under the BSM can be priced more easily via a single Laplace inversion). Furthermore, our algorithm runs very fast under the BSM and DEM (the CPU times to generate one numerical result under the BSM and DEM are around 0.1 seconds and 1.7 seconds, respectively). To save space, we omit the corresponding numerical tables, but they are available on request.

It is worth mentioning that the Monte Carlo simulation estimates listed here, which serve only as a benchmark, are obtained via an (almost) plain Monte Carlo simulation scheme along with the variance reduction technique of control variates. For more variance reduction techniques of the Monte Carlo simulation in financial engineering, we refer the reader to the monograph by Glasserman [20].

In addition, Kwok and Lau (see, [25, p. 10]) provided one numerical result (around 9.18, with an associated parameter setting of $\alpha=0.8, r=0.05, \sigma=0.2, S_{0}=100, K=95$, and $t=0.25$ ) using a forward shooting grid method, whereas applying our Euler inversion method yields 9.1873. The coincidence also implies the correctness of our method. 
TABLE 2: Hedging parameters of fixed- and floating-strike quantile options. The default parameters are $\lambda=3, r=0.05, \eta_{1}=\theta_{1}=30, \eta_{2}=\theta_{2}=40, p_{1}=p_{2}=0.3, q_{1}=q_{2}=0.2, S_{0}=100, t=1$, $A_{1}=A_{2}=18, n_{1}=n_{2}=90$, and $X=10000$. LL deltas and LL gammas are obtained by using Laplace inversion twice. MC deltas and $\mathrm{MC}$ gammas are the Monte Carlo simulation estimates obtained by simulating 100000 paths and using 100000 time steps. SE is the associated standard error.

\begin{tabular}{|c|c|c|c|c|c|c|c|}
\hline \multicolumn{8}{|c|}{ Deltas of fixed-strike quantile options under the HEM } \\
\hline \multirow{2}{*}{$\sigma$} & \multirow{2}{*}{$K$} & \multicolumn{3}{|c|}{$\alpha=0.3$} & \multicolumn{3}{|c|}{$\alpha=0.6$} \\
\hline & & LL delta & MC delta & SE & LL delta & MC delta & SE \\
\hline \multirow[t]{3}{*}{0.2} & 90 & 0.72119 & 0.72003 & 0.00077 & 0.89953 & 0.89899 & 0.00070 \\
\hline & 100 & 0.42080 & 0.42219 & 0.00097 & 0.64966 & 0.65052 & 0.00097 \\
\hline & 110 & 0.14637 & 0.14724 & 0.00091 & 0.34885 & 0.34956 & 0.00097 \\
\hline \multirow[t]{2}{*}{0.3} & 90 & 0.61653 & 0.61651 & 0.00080 & 0.84106 & 0.84073 & 0.00080 \\
\hline & 100 & 0.39535 & 0.39668 & 0.00096 & 0.63839 & 0.63767 & 0.00096 \\
\hline \multicolumn{8}{|c|}{ Deltas of floating-strike quantile options under the HEM } \\
\hline \multirow{2}{*}{$\sigma$} & \multirow{2}{*}{$K$} & \multicolumn{3}{|c|}{$\alpha=0.3$} & \multicolumn{3}{|c|}{$\alpha=0.6$} \\
\hline & & LL delta & MC delta & SE & LL delta & MC delta & SE \\
\hline 0.2 & - & 0.01930 & 0.01918 & 0.00012 & 0.04676 & 0.04666 & 0.00020 \\
\hline 0.3 & - & 0.02978 & 0.02964 & 0.00017 & 0.07284 & 0.07274 & 0.00029 \\
\hline \multicolumn{8}{|c|}{ Gammas of fixed-strike quantile options under the HEM } \\
\hline \multirow{2}{*}{$\sigma$} & \multirow{2}{*}{$K$} & \multicolumn{3}{|c|}{$\alpha=0.3$} & \multicolumn{3}{|c|}{$\alpha=0.6$} \\
\hline & & LL gamma & MC gamma & SE & LL gamma & MC gamma & SE \\
\hline \multirow[t]{3}{*}{0.2} & 90 & 0.01992 & 0.02079 & 0.00157 & 0.01363 & 0.01243 & 0.00121 \\
\hline & 100 & 0.03647 & 0.03924 & 0.00225 & 0.03432 & 0.03531 & 0.00215 \\
\hline & 110 & 0.01956 & 0.02028 & 0.00168 & 0.02681 & 0.02821 & 0.00201 \\
\hline \multirow[t]{3}{*}{0.3} & 90 & 0.01715 & 0.01770 & 0.00143 & 0.01403 & 0.01275 & 0.00119 \\
\hline & 100 & 0.02429 & 0.02783 & 0.00188 & 0.02424 & 0.02559 & 0.00179 \\
\hline & 110 & 0.01649 & 0.01548 & 0.00144 & 0.02069 & 0.02207 & 0.00177 \\
\hline
\end{tabular}

\subsection{Sensitivity of our numerical algorithm to $A_{1}, A_{2}$, and $X$}

Observe that the Euler inversion algorithm depends on the selection of parameters $A_{1}, A_{2}$, and $X$, all of which are primarily for discretization error control. As a matter of fact, the algorithm is quite insensitive to changes in $A_{1}, A_{2}$, and $X$. This property is well illustrated by Figure 1, where the upper three graphs show how the absolute errors between the LL prices and MC prices change as $A_{1}, A_{2}$, and $X$ vary; while the lower three graphs show the corresponding relative errors. Actually, the LL prices keep accurate and stable when these parameters vary in broad regions, i.e. $A_{1} \in[15,45], A_{2} \in[15,45]$, and $X \in[1000,30000]$. The absolute values of associated relative errors between LL prices and MC prices are stable and stay smaller than $0.06 \%$. Consequently, we draw the conclusion that our pricing algorithm is stable and, thus, reliable.

\subsection{Convergence and computational cost of our numerical algorithm}

Recall that our numerical algorithm involves four algorithm parameters, $n_{1}$ (and $n_{2}$, typically we select $n_{2}=n_{1}$ in practice), $A_{1}, A_{2}$, and $X$. As discussed in Section 4.1, $n_{1}$ (and $\left.n_{2}\right)$ is 

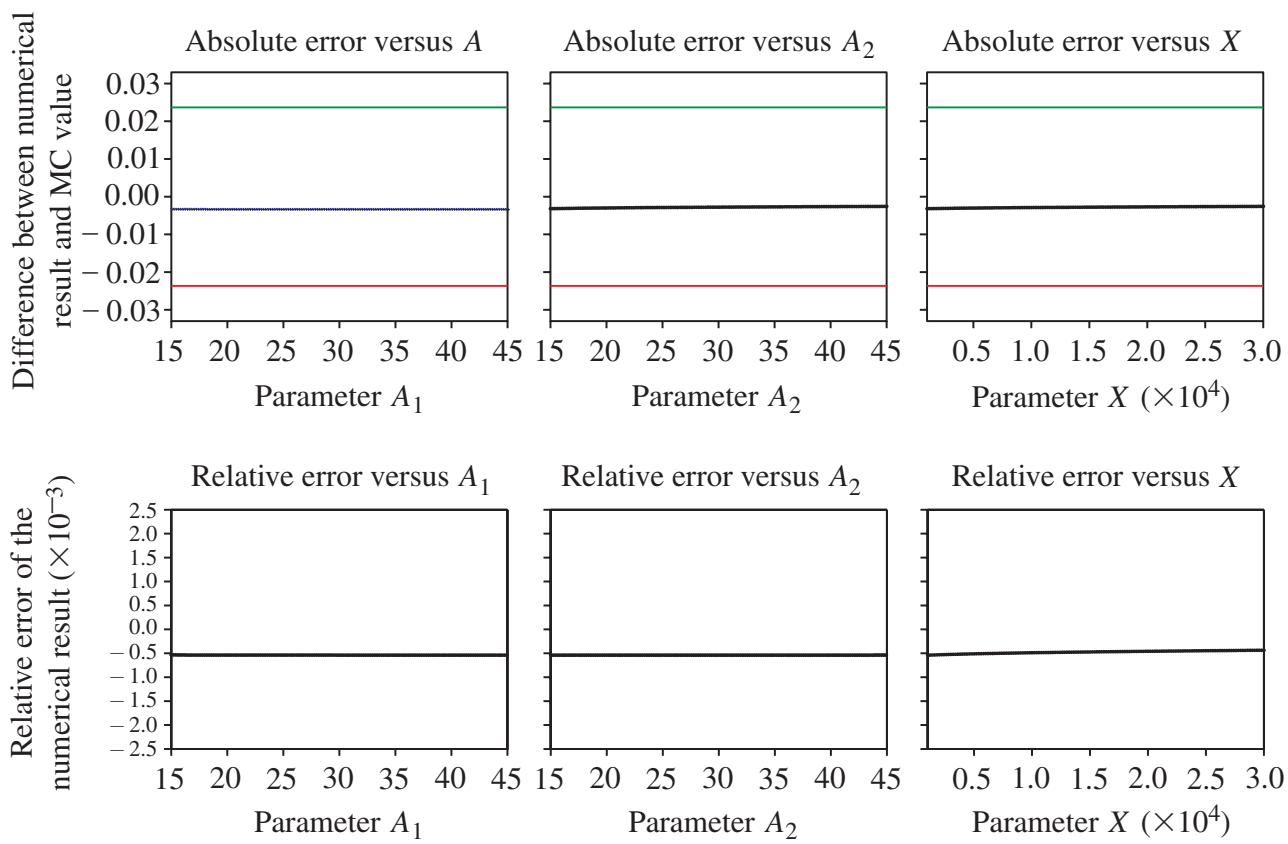

Relative error versus $X$

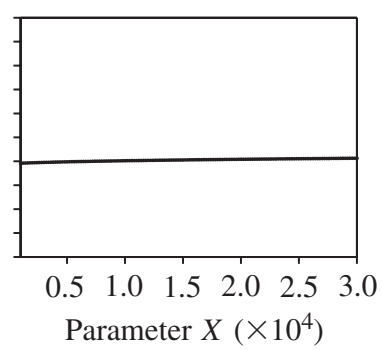

Figure 1: How the numerical results (LL prices) change as $A_{1}, A_{2}$, and $X$ vary. The parameter setting is $\alpha=0.5, r=0.05, \sigma=0.2, \lambda=3, \eta_{1}=\theta_{1}=30, \eta_{2}=\theta_{2}=40, p_{1}=p_{2}=0.3, q_{1}=q_{2}=0.2$, $S_{0}=100, t=1$, and $n_{1}=n_{2}=90$. Upper-left graph: absolute error versus $A_{1}$, where $A_{2}=18$ and $X=10000$. Upper-middle graph: absolute error versus $A_{2}$, where $A_{1}=18$ and $X=10000$. Upper-right graph: absolute error versus $X$, where $A_{1}=18$ and $A_{2}=18$. The lower three graphs show how the corresponding relative errors change.

related to the approximation to the infinite series in the inversion formula (4.1) by some finite sum called the Euler transformation (see [1]). More precisely, applying the Euler transformation to accelerate the rate of the convergence of a series, we approximate the infinite series of the form $\sum_{i=0}^{\infty}(-1)^{i} a_{i}$ in the inversion formula (4.1) by the finite sum

$$
E\left(m_{1}, n_{1}\right)=\sum_{k=0}^{m_{1}} \frac{m_{1} !}{k !\left(m_{1}-k\right) !} 2^{-m_{1}} S_{n_{1}+k},
$$

where $m_{1}=n_{1}+15$ (suggested by Abate and Whitt [1]) and $S_{j}:=\sum_{i=0}^{j}(-1)^{i} a_{i}$ for $j=$ $0,1, \ldots$ It is easily seen that the finite sum $E\left(m_{1}, n_{1}\right)$ involves only the first $m_{1}+n_{1}=2 n_{1}+15$ terms of the series. Therefore, the larger $n_{1}$, the more accurate the approximation. As $n_{1}$ goes to $\infty$, the approximation converges to the sum of the infinite series.

In this section we intend to study how our numerical algorithm converges as $n_{1}$ increases, given $A_{1}, A_{2}$, and $X$. For ease of exposition, we denote by $p\left(n_{1}\right)$ the numerical quantile option price obtained via our numerical algorithm with parameter $n_{1}$. Let $n_{1}$ increase from 15 with an increment of 5 , i.e. $n_{1}=15,20,25,30, \ldots$ It is worth noting that we do not know the true option price, i.e. the limit of the numerical approximation as $n_{1}$ goes to $\infty$. Alternatively, we may use $\left|p\left(n_{1}+5\right)-p\left(n_{1}\right)\right|$ to measure the convergence, and say that the numerical approximation is sufficiently close to its limit when

$$
\left|p\left(n_{1}+5\right)-p\left(n_{1}\right)\right|<10^{-4} .
$$


TABLE 3: How the numerical prices $p\left(n_{1}\right)$ converge and how the CPU times (in seconds) change as $n_{1}$ increases. The default parameters are $\alpha=0.5, r=0.05, \eta_{1}=\theta_{1}=30, \eta_{2}=\theta_{2}=40, p_{1}=p_{2}=0.3$, $q_{1}=q_{2}=0.2, S_{0}=100, t=1, A_{1}=A_{2}=18$, and $X=10000$.

\begin{tabular}{rccccccc}
\hline & & & CPU & & & CPU \\
$n_{1}$ & $p\left(n_{1}\right)$ & $\left|p\left(n_{1}+5\right)-p\left(n_{1}\right)\right|$ & time & $n_{1}$ & $p\left(n_{1}\right)$ & $\left|p\left(n_{1}+5\right)-p\left(n_{1}\right)\right|$ & time \\
\hline 20 & 5.92142 & 0.007252 & 1.73 & 115 & 5.90396 & 0.000020 & 31.97 \\
25 & 5.91417 & 0.003769 & 2.36 & 120 & 5.90394 & 0.000017 & 34.56 \\
30 & 5.91040 & 0.002146 & 3.11 & 125 & 5.90392 & 0.000014 & 37.16 \\
35 & 5.90826 & 0.001310 & 3.97 & 130 & 5.90391 & 0.000012 & 40.02 \\
40 & 5.90695 & 0.000844 & 4.98 & 135 & 5.90390 & 0.000011 & 43.08 \\
45 & 5.90610 & 0.000568 & 6.05 & 140 & 5.90389 & 0.000009 & 46.11 \\
50 & 5.90554 & 0.000397 & 7.19 & 145 & 5.90388 & 0.000008 & 49.36 \\
55 & 5.90514 & 0.000285 & 8.48 & 150 & 5.90387 & 0.000007 & 52.55 \\
60 & 5.90485 & 0.000210 & 9.86 & 155 & 5.90386 & 0.000006 & 56.11 \\
65 & 5.90464 & 0.000158 & 11.34 & 160 & 5.90386 & 0.000006 & 59.73 \\
70 & 5.90448 & 0.000121 & 12.98 & 165 & 5.90385 & 0.000005 & 63.16 \\
75 & 5.90436 & 0.000095 & 14.67 & 170 & 5.90384 & 0.000004 & 67.00 \\
80 & 5.90427 & 0.000075 & 16.48 & 175 & 5.90384 & 0.000004 & 71.02 \\
85 & 5.90420 & 0.000060 & 18.41 & 180 & 5.90384 & 0.000004 & 75.02 \\
90 & 5.90413 & 0.000049 & 20.39 & 185 & 5.90383 & 0.000003 & 78.97 \\
95 & 5.90408 & 0.000040 & 22.50 & 190 & 5.90383 & 0.000003 & 82.81 \\
100 & 5.90404 & 0.000033 & 24.97 & 195 & 5.90383 & 0.000003 & 87.39 \\
105 & 5.90401 & 0.000028 & 27.02 & 200 & 5.90382 & - & 91.80 \\
\hline
\end{tabular}
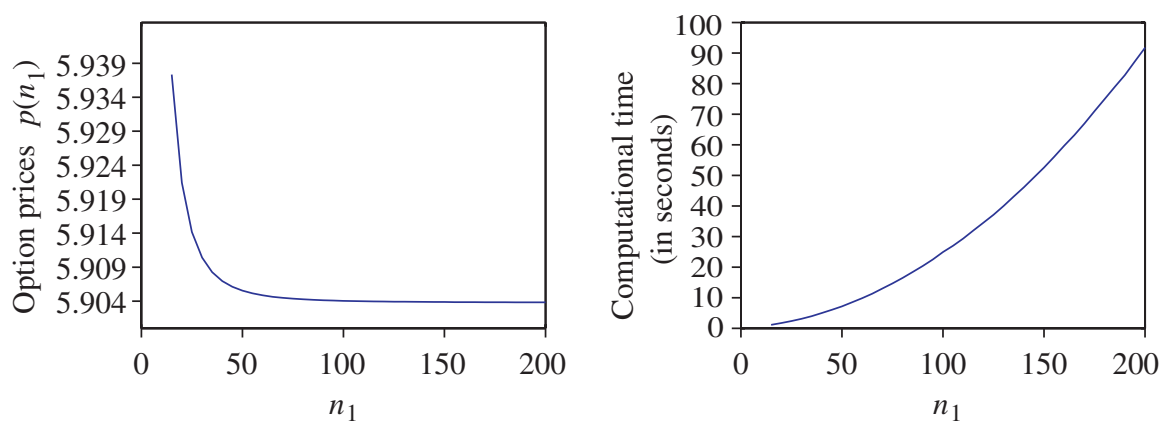

FIGURE 2: The left panel and the right panel illustrate how the numerical prices converge and how the computational times change as $n_{1}\left(=n_{2}\right)$ increases, respectively. We can see that the numerical prices converge quite fast as $n_{1}$ increases. The parameter setting is $\alpha=0.5, r=0.05, \sigma=0.2, \lambda=3$, $\eta_{1}=\theta_{1}=30, \eta_{2}=\theta_{2}=40, p_{1}=p_{2}=0.3, q_{1}=q_{2}=0.2, S_{0}=100, t=1, A_{1}=A_{2}=18$, and $X=10000$.

It turns out that the $n_{1}$ s that satisfy (5.1) can guarantee three-digit accuracy of our numerical prices.

The results of Table 3 demonstrate how the numerical prices $p\left(n_{1}\right)$ converge as $n_{1}$ increases (see also the left panel of Figure 2). It can be seen that when $n_{1} \geq 75$, we have $\mid p\left(n_{1}+5\right)-$ $p\left(n_{1}\right) \mid<10^{-4}$. Furthermore, this can guarantee at least three-digit accuracy (5.904) for our numerical prices if we round them off to three decimal places. This accuracy is acceptable in practice because of the bid-ask spreads. In order to be safe, we select $n_{1}=90$ when implementing the algorithm in our numerical part (Section 5.1). In addition, the results of 
Table 3 also demonstrate how the CPU times change as $n_{1}$ increases (see also the right panel of Figure 2). When $n_{1}=90$, the corresponding CPU time is around 21 seconds and practically acceptable.

Note that the above numerical experiments are conducted when $A_{1}=18, A_{2}=18$, and $X=10000$ are fixed. However, the convergence speed of our numerical algorithm may also depend on selection of these three algorithm parameters. This issue will be investigated in Section 5.4.

\subsection{Effect of $A_{1}, A_{2}$, and $X$ on the convergence speed of our numerical algorithm}

To study the effect of the selection of the three parameters $A_{1}, A_{2}$, and $X$ on the convergence speed of our numerical algorithm, we define $N^{*}\left(A_{1}, A_{2}, X\right)$ for given $A_{1}, A_{2}$, and $X$ as

$$
N^{*}\left(A_{1}, A_{2}, X\right):=\min \left\{n_{1}:\left|p\left(n_{1}+5\right)-p\left(n_{1}\right)\right|<10^{-4} \text { and } n_{1}=15,20,25, \ldots\right\} .
$$

Note that the smaller $N^{*}\left(A_{1}, A_{2}, X\right)$, the faster the convergence. Accordingly, $N^{*}\left(A_{1}, A_{2}, X\right)$ may be used to indicate the convergence speed corresponding to the parameter selection, $A_{1}$, $A_{2}$, and $X$. As discussed in Section 5.3, the $n_{1}$ s that satisfy $\left|p\left(n_{1}+5\right)-p\left(n_{1}\right)\right|<10^{-4}$ can typically achieve at least three-digit accuracy for our numerical prices if we round them off to three decimal places. This accuracy is acceptable in practice because of the bid-ask spreads.

By plotting $N^{*}\left(A_{1}, A_{2}, X\right)$ for various $A_{1}, A_{2}$, and $X$, Figure 3 illustrates how these three parameters influence the convergence speed of our numerical algorithm. The upper three graphs
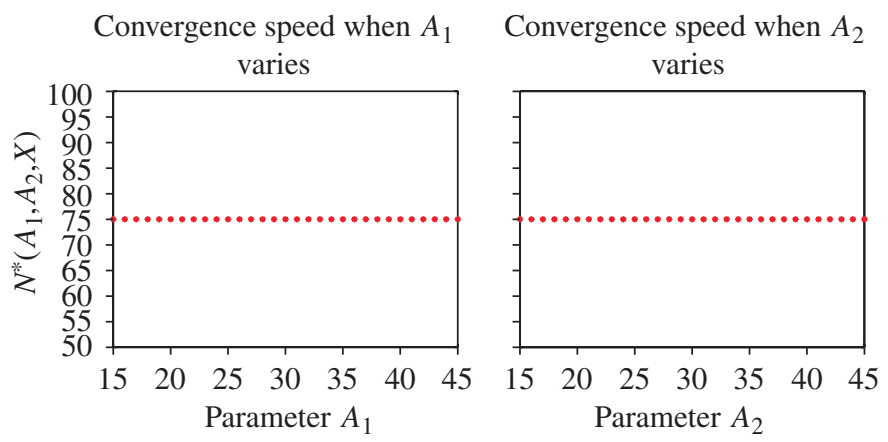

Convergence speed when $X$

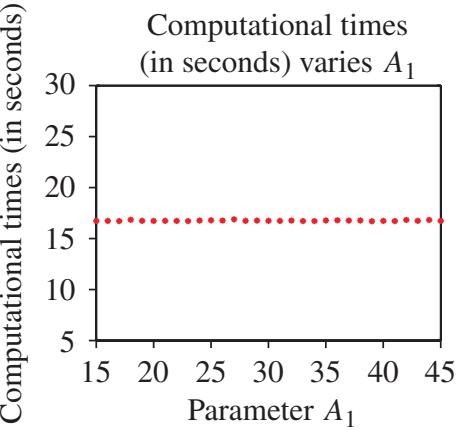

Computational times

(in seconds) varies $A_{2}$

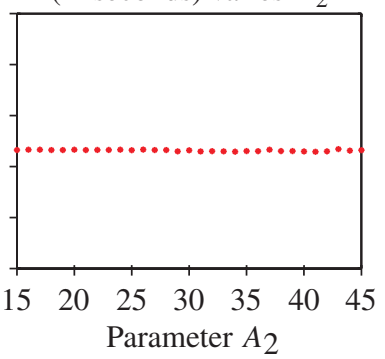
varies

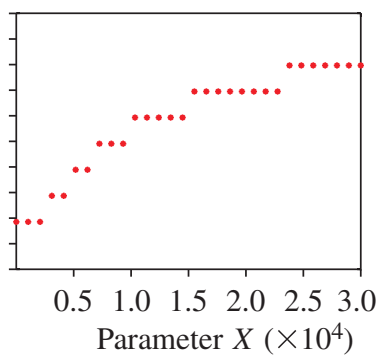

Computational times

(in seconds) varies $X$

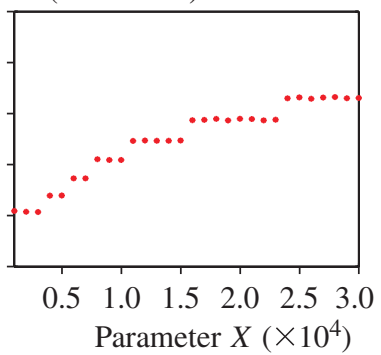

FIGURE 3: The effect of the selection of the three parameters $A_{1}, A_{2}$, and $X$ on the convergence speed of our numerical algorithm. The parameter setting is $\alpha=0.5, r=0.05, \sigma=0.2, \lambda=3, \eta_{1}=\theta_{1}=30$, $\eta_{2}=\theta_{2}=40, p_{1}=p_{2}=0.3, q_{1}=q_{2}=0.2, S_{0}=100$, and $t=1$. Upper-left graph: $N^{*}\left(A_{1}, A_{2}, X\right)$ versus $A_{1}$ when $A_{2}=18$ and $X=10000$. Upper-middle graph: $N^{*}\left(A_{1}, A_{2}, X\right)$ versus $A_{2}$ when $A_{1}=18$ and $X=10000$. Upper-right graph: $N^{*}\left(A_{1}, A_{2}, X\right)$ versus $X$ when $A_{1}=18$ and $A_{2}=18$.

The lower three graphs show how the corresponding computational times change. 
show how $N^{*}\left(A_{1}, A_{2}, X\right)$ changes when $A_{1}, A_{2}$, and $X$ vary, respectively, whereas the lower three exhibit the corresponding computational times. We can see that the convergence speed of our numerical algorithm seems insensitive to the selection of $A_{1}$ and $A_{2}$ because broad regions of $A_{1}$ and $A_{2}\left(A_{1} \in[15,45], A_{2} \in[15,45]\right)$ can guarantee the same $N^{*}\left(A_{1}, A_{2}, X\right)$ $(=75)$. In contrast, the selection of parameter $X$ has a larger effect on the convergence speed. The upper-right graph demonstrates that a larger $X$ tends to result in a larger $N^{*}\left(A_{1}, A_{2}, X\right)$, i.e. a slower convergence. However, a broad region of $X(X \in[1000,30000])$ can guarantee $N^{*}\left(A_{1}, A_{2}, X\right) \leq 90$. Recall that to be safe, we select $n_{1}=90$ when implementing the numerical algorithm in our numerical part (Section 5.1). Figure 3 indicates that this selection of $n_{1}$ is quite good because it can guarantee $\left|p\left(n_{1}+5\right)-p\left(n_{1}\right)\right| \leq 10^{-4}$ for broad regions of $A_{1}, A_{2}$, and $X\left(A_{1} \in[15,45], A_{2} \in[15,45]\right.$, and $\left.X \in[1000,30000]\right)$.

\section{Appendix A. Proof of Theorem 3.1}

Result (3.1) can be derived as follows:

$$
\begin{aligned}
\mathcal{L}(v) & =\int_{-\infty}^{+\infty} \mathrm{e}^{-v k} X \mathrm{E}\left[\left(\frac{S_{0}}{X} \mathrm{e}^{Q(\alpha, t, X)}-\mathrm{e}^{-k}\right)^{+}\right] \mathrm{d} k \\
& =X \mathrm{E}\left[\int_{-\infty}^{+\infty} \mathrm{e}^{-v k}\left[\left(\frac{S_{0}}{X} \mathrm{e}^{Q(\alpha, t, X)}-\mathrm{e}^{-k}\right)^{+}\right] \mathrm{d} k\right] \\
& =X \mathrm{E}\left[\int_{-\log \left(S_{0} / X\right)-Q(\alpha, t, X)}^{+\infty} \mathrm{e}^{-v k}\left(\frac{S_{0}}{X} \mathrm{e}^{Q(\alpha, t, X)}-\mathrm{e}^{-k}\right) \mathrm{d} k\right] \\
& =\frac{S_{0}^{v+1}}{v(v+1) X^{v}} \mathrm{E}\left[\mathrm{e}^{(v+1) Q(\alpha, t, X)}\right] .
\end{aligned}
$$

In the light of Proposition 2.2, we have

$$
\mathrm{E}\left[\mathrm{e}^{(v+1) Q(\alpha, t, X)}\right]=\mathrm{E}\left[\mathrm{e}^{(v+1) M_{X}(\alpha t)}\right] \mathrm{E}\left[\mathrm{e}^{(v+1) m_{X}((1-\alpha) t)}\right] .
$$

Then (3.1) follows immediately. Note that, under the BSM, the moment generating function of the running maximum of the drifted Brownian motion during a period can be expressed explicitly with complementary error functions (or error functions). By slightly modifying the formula on page 250 of [5], we can obtain (3.2) easily. Finally, the two Greeks can be obtained by interchanging derivatives and integrals based on Theorem A.12 of [31, pp. 203-204].

\section{Appendix B. Proof of Theorem 3.2}

Note that the floating-strike $\alpha$-quantile option price can be rewritten as

$$
\begin{aligned}
P_{\alpha}\left(S_{0}, T, \S\right) & =\mathrm{e}^{-r T} \mathrm{E}\left[(Q(\alpha, T, \S)-S(T))^{+}\right] \\
& =\mathrm{E}\left[\frac{S(T)}{S_{0}} \mathrm{e}^{-r T}\left(\frac{S_{0}}{S(T)} Q(\alpha, T, \S)-S_{0}\right)^{+}\right] .
\end{aligned}
$$

Consider introducing a new measure $\widetilde{\mathrm{P}}$ defined as

$$
\left.\frac{\mathrm{d} \widetilde{\mathrm{P}}}{\mathrm{dP}}\right|_{t=T}=\frac{S(T)}{S_{0}} \mathrm{e}^{-(r-q) T} .
$$


Then according to Girsanov's theorem for jump diffusion processes (see [32]), we conclude that, under the new measure $\widetilde{\mathrm{P}},\{\widetilde{W}(t):=W(t)-\sigma t: 0 \leq t \leq T\}$ becomes a standard Brownian motion, and the original return process

$$
\begin{aligned}
X(t) & =\left(r-q-\frac{\sigma^{2}}{2}-\lambda \zeta\right) t+\sigma W(t)+\sum_{i=1}^{N(t)} Y_{i} \\
& =\left(r-q+\frac{\sigma^{2}}{2}-\lambda \zeta\right) t+\sigma \widetilde{W}(t)+\sum_{i=1}^{\tilde{N}(t)} \widetilde{Y}_{i}
\end{aligned}
$$

is a new hyperexponential jump diffusion process, where $\{\tilde{N}(t): t \geq 0\}$ is a Poisson process with rate $\widetilde{\lambda}=\lambda \mathrm{E}\left[\mathrm{e}^{Y}\right]=\lambda(\zeta+1)$, and $\left\{\widetilde{Y}_{i}: i=1,2, \ldots\right\}$ is also a sequence of i.i.d. hyperexponential random variables with PDF

$$
\begin{aligned}
f_{\widetilde{Y}}(x)= & f_{Y}(x) \frac{\mathrm{e}^{x}}{\mathrm{E}\left[\mathrm{e}^{Y}\right]} \\
= & \sum_{i=1}^{m} \frac{p_{i} \eta_{i}}{(\zeta+1)\left(\eta_{i}-1\right)}\left(\eta_{i}-1\right) \mathrm{e}^{-\left(\eta_{i}-1\right) x} \mathbf{1}_{\{x \geq 0\}} \\
& +\sum_{j=1}^{n} \frac{q_{j} \theta_{j}}{(\zeta+1)\left(\theta_{j}+1\right)}\left(\theta_{j}+1\right) \mathrm{e}^{\left(\theta_{j}+1\right) x} \mathbf{1}_{\{x<0\}} \\
= & : \sum_{i=1}^{n} \widetilde{p}_{i} \tilde{\eta}_{i} \mathrm{e}^{-\tilde{\eta}_{i} x} \mathbf{1}_{\{x \geq 0\}}+\sum_{j=1}^{m} \widetilde{q}_{j} \tilde{\theta}_{j} \mathrm{e}^{\tilde{\theta}_{j} x} \mathbf{1}_{\{x<0\}} .
\end{aligned}
$$

Here

$$
\begin{aligned}
& \tilde{p}_{i}:=\frac{p_{i} \eta_{i}}{(\zeta+1)\left(\eta_{i}-1\right)}>0 \quad \text { and } \quad \tilde{\eta}_{i}:=\eta_{i}-1>1 \quad \text { for all } i=1, \ldots, m, \\
& \tilde{q}_{j}:=\frac{q_{j} \theta_{j}}{(\zeta+1)\left(\theta_{j}+1\right)}>0 \quad \text { and } \quad \tilde{\theta}_{j}:=\theta_{j}+1>1 \quad \text { for all } j=1, \ldots, n .
\end{aligned}
$$

By the change of measure, (B.1) is reduced to

$$
P_{\alpha}\left(S_{0}, T, \delta\right)=\mathrm{e}^{-q T} \widetilde{\mathrm{E}}\left[\left(\frac{S_{0}}{S(T)} Q(\alpha, T, \delta)-S_{0}\right)^{+}\right] .
$$

A close scrutiny of $S_{0} Q(\alpha, T, \S) / S(T)$ (see [16] or [26]) results in

$$
\begin{aligned}
\frac{S_{0}}{S(T)} Q(\alpha, T, \delta) & =\frac{S_{0}}{S(T)} \inf \left\{x \in \mathbb{R}: \int_{0}^{T} \mathbf{1}_{\{S(t) \leq x\}} \mathrm{d} t>\alpha T\right\} \\
& =\inf \left\{\frac{S_{0}}{S(T)} x \in \mathbb{R}: \int_{0}^{T} \mathbf{1}_{\left\{S_{0} S(t) / S(T) \leq S_{0} x / S(T)\right\}} \mathrm{d} t>\alpha T\right\} \\
& =\inf \left\{z \in \mathbb{R}: \int_{0}^{T} \mathbf{1}_{\left\{S_{0} S(t) / S(T) \leq z\right\}} \mathrm{d} t>\alpha T\right\} .
\end{aligned}
$$


Note that, for any $t \in[0, T]$,

$$
\begin{aligned}
\frac{S_{0}}{S(T)} S(t)=S_{0} \exp ( & \left.\left(r-q+\frac{\sigma^{2}}{2}-\lambda \zeta\right)(t-T)+\sigma(\widetilde{W}(t)-\widetilde{W}(T))-\sum_{i=\widetilde{N}(t)+1}^{\widetilde{N}(T)} \tilde{Y}_{i}\right) \\
=S_{0} \exp ( & \left(q-r-\frac{\sigma^{2}}{2}+\lambda \zeta\right)(T-t)+\sigma[(-\tilde{W}(T))-(-\widetilde{W}(t))] \\
& \left.+\sum_{i=\widetilde{N}(t)+1}^{\tilde{N}(T)}\left(-\tilde{Y}_{i}\right)\right) .
\end{aligned}
$$

Introduce a new standard Brownian motion $\{\bar{W}(t):=-\widetilde{W}(t): 0 \leq t \leq T\}$, and define $\bar{Y}_{i}:=$ $-\widetilde{Y}_{i}$ and $\bar{S}(t):=S_{0} \exp \left(\bar{\mu} t+\sigma \bar{W}(t)+\sum_{i=1}^{\widetilde{N}(t)} \bar{Y}_{i}\right)$, where $\bar{\mu}:=\bar{r}-\bar{q}-\sigma^{2} / 2-\tilde{\lambda} \bar{\zeta}, \bar{r}:=q$, $\bar{q}:=r-\lambda \zeta-\tilde{\lambda} \bar{\zeta}$, and $\bar{\zeta}:=\mathrm{Ee}^{\bar{Y}}-1$. Then we claim that, under the new measure $\widetilde{\mathrm{P}}$, the following two processes have the same distribution:

$$
\left\{\frac{S_{0}}{S(T)} S(t): t \in[0, T]\right\} \stackrel{\mathrm{D}}{=}\{\bar{S}(T-t): t \in[0, T]\} .
$$

Consequently, we know from (B.3) and (B.4) that, under the new measure $\widetilde{P}$,

$$
\begin{aligned}
\frac{S_{0}}{S(t)} Q(\alpha, T, \delta) & =\inf \left\{z \in \mathbb{R}: \int_{0}^{T} \mathbf{1}_{\{S S(t) / S(T) \leq z\}} \mathrm{d} t>\alpha T\right\} \\
& \stackrel{\mathrm{D}}{=} \inf \left\{z \in \mathbb{R}: \int_{0}^{T} \mathbf{1}_{\{\bar{S}(T-t) \leq z\}} \mathrm{d} t>\alpha T\right\} \\
& =\inf \left\{z \in \mathbb{R}: \int_{0}^{T} \mathbf{1}_{\{\bar{S}(t) \leq z\}} \mathrm{d} t>\alpha T\right\} \\
& =Q(\alpha, T, \bar{\delta}) .
\end{aligned}
$$

From (B.2) and (B.5), (3.3) follows immediately. For the two Greeks, noting that

$$
P_{\alpha}\left(S_{0}, T, \delta\right)=\mathrm{e}^{-r T} \mathrm{E}\left[(Q(\alpha, T, \&)-S(T))^{+}\right]=\mathrm{e}^{-r T} \mathrm{E}\left[\left(\mathrm{e}^{Q(\alpha, T, X)}-\mathrm{e}^{X(T)}\right)^{+}\right] S_{0},
$$

we easily obtain

$$
\Gamma\left(P_{\alpha}\left(S_{0}, T, \S\right)\right)=0
$$

and

$$
\Delta\left(P_{\alpha}\left(S_{0}, T, \S\right)\right)=\mathrm{e}^{-r T} \mathrm{E}\left[\left(\mathrm{e}^{Q(\alpha, T, X)}-\mathrm{e}^{X(T)}\right)^{+}\right]=P_{\alpha}(1, T, \S)=C_{\alpha}(1,1, T, \bar{\jmath}) .
$$

The proof is completed.

\section{Acknowledgements}

The author is grateful to Professor S. G. Kou of Columbia University, who brought this topic to his attention. Moreover, the author would also like to thank an anonymous referee, the Associate Editor, and the Editor for many helpful comments and to thank Professor Jiro Akahori of Ritsumeikan University, Professor Angelos Dassios of the London School of Economics and Political Science, Professor Yue-Kuen Kwok of Hong Kong University of Science 
and Technology, and Professor Jeremy Staum of Northwestern University for their valuable suggestions. In addition, the author is grateful for the support from the General Research Fund (GRF) of Research Grants Council of Hong Kong (project reference number 610709) and Hong Kong RGC Direct Allocation Grant (2008/2009) (project number DAG08/09.EG07).

\section{References}

[1] Aваte, J. And Whitt, W. (1992). The Fourier-series method for inverting transforms of probability distributions. Queueing Systems 10, 5-87.

[2] Akahori, J. (1995). Some formulae for a new type of path-dependent option. Ann. Appl. Prob. 5, 383-388.

[3] Asmussen, S., Avram, F. and Pistorius, M. R. (2004). Russian and American put options under exponential phase-type Lévy models. Stoch. Process. Appl. 109, 79-111.

[4] Bertoin, J., Chaumont, L. And Yor, M. (1997). Two chain transformations and their applications to quantiles. J. Appl. Prob. 34, 882-897.

[5] Borodin, A. N. And Salminen P. (2002). Handbook of Brownian Motion-Facts and Formulae, 2nd edn. Birkhäuser, Basel.

[6] Broadie, M. and Detemple, J. B. (2004). Option pricing: valuation models and applications. Manag. Sci. 50, $1145-1177$.

[7] CaI, N. (2009). On first passage times of a hyper-exponential jump diffusion process. Operat. Res. Lett. 37, $127-134$.

[8] CAI, N. And Kou, S. G. (2011). Option pricing under a mixed-exponential jump diffusion model. To appear in Mamag. Sci.

[9] CaI, N., Chen, N. And Wan, X. (2010). Occupation times of jump-diffusion processes with double exponential jumps and the pricing of options. Math. Operat. Res. 35, 412-437.

[10] Carr, P. and Madan, D. B. (1999). Option valuation using the fast Fourier transform. J. Comput. Finance 2, 61-73.

[11] Choudhury, G. L., Lucantoni, D. M. and Whitt, W. (1994). Multidimensional transform inversion with applications to the transient M/G/1 queue. Ann. Appl. Prob. 4, 719-740.

[12] Craddock, M., Heath, D. and Platen, E. (2000). Numerical inversion of Laplace transforms: a survey of techniques with applications to derivative pricing. J. Comput. Finance 4, 57-81.

[13] Dassios, A. (1995). The distribution of the quantile of a Brownian motion with drift and the pricing of related path-dependent options. Ann. Appl. Prob. 5, 389-398.

[14] Dassios, A. (1996). Sample quantiles of stochastic processes with stationary and independent increments. Ann. Appl. Prob. 6, 1041-1043.

[15] Davydov, D. And Linetsky, V. (2001). Structuring, pricing and hedging double barrier step options. J. Comput. Finance 5, 55-87.

[16] Detemple, J. (2001). American options: symmetry properties. In Option Pricing, Interest Rates and Risk Management, eds E. Jouini, J. Cvitanic and M. Musiela, Cambridge University Press, pp. 67-104.

[17] Embrechts, P., Rogers, L. C. G. AND Yor, M. (1995). A proof of Dassios' representation of the $\alpha$-quantile of Brownian motion with drift. Ann. Appl. Prob. 5, 757-767.

[18] Fusai, G. (2000). Corridor options and arc-sine law. Ann. Appl. Prob. 10, 634-663.

[19] Fusai, G. and Tagliani, A. (2001). Pricing of occupation time derivatives: continuous and discrete monitoring. J. Comput. Finance 5, 1-37.

[20] Glasserman, P. (2000). Monte Carlo Methods in Financial Engineering. Springer, New York.

[21] Hugonnier, J. (1999). The Feynman-Kac formula and pricing occupation time derivatives. Internat. J. Theoret. Appl. Finance 2, 153-178.

[22] Jeannin, M. And Pistorious, M. (2010). A transform approach to compute prices and Greeks of barrier options driven by a class of Lévy processes. Quant. Finance 10, 629-644.

[23] Kou, S. G. (2002). A jump-diffusion model for option pricing. Manag. Sci. 48, 1086-1101.

[24] KwoK, Y. K. (I998). Mathematical Models of Financial Derivatives. Springer, Singapore.

[25] KwoK, Y. K. And LaU, K. W. (2001). Pricing algorithms for options with exotic path-dependence. J. Derivatives 9, 23-38.

[26] Leung, K. S. AND KwoK, Y. K. (2007). Distribution of occupation times for constant elasticity of variance diffusion and the pricing of $\alpha$-quantile options. Quant. Finance 7, 87-94.

[27] Linetsky, V. (1999). Step options. Math. Finance 9, 55-96.

[28] Miura, R. (1992). A note on look-back options based on order statistics. Hitotsubashi J. Commerce Manag. 27, 15-28.

[29] Pechtl, A. (1999). Some applications of occupation times of Brownian motion with drift in mathematical finance. J. Appl. Math. Decision Sci. 3, 63-73. 
[30] Petrella, G. (2004). An extension of the Euler Laplace transform inversion algorithm with applications in option pricing. Operat. Res. Lett. 32, 380-389.

[31] Schiff, J. L. (1999). The Laplace Transform. Springer, New York.

[32] Schoroder, M. (1999). Changes of numeraire for pricing futures, forwards, and options. Rev. Financial Studies 12, 1143-1163.

[33] Shreve, S. E. (2004). Stochastic Calculus for Finance. II. Springer, New York.

[34] Yor, M. (1995). The distribution of Brownian quantiles. J. Appl. Prob. 32, 405-416. 